\title{
Doença Metabólica Óssea da Prematuridade em \\ Recém-Nascidos de Muito Baixo Peso: Estudo \\ Observacional Retrospetivo
}

\section{Metabolic Bone Disease of Prematurity in Very Low Birthweight Infants: Retrospective Observational Study}

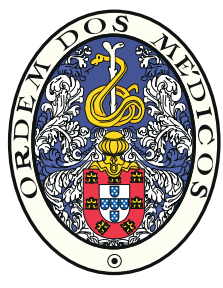

\author{
Raquel COSTA $\triangle^{1}$, Catarina FRANCO ${ }^{2}$, Nádia SANTOS ${ }^{1}$, Patrícia MAIO ${ }^{1}$, Filipa VIEIRA ${ }^{3}$, Sónia ANTUNES ${ }^{4}$, \\ Laura MARTINS ${ }^{4}$, Madalena Lopo TUNA ${ }^{5}$ \\ Acta Med Port 2019 Jul-Aug;32(7-8):536-541 - https://doi.org/10.20344/amp.10994
}

\section{RESUMO}

Introdução: A doença metabólica óssea da prematuridade consiste numa diminuição da matriz óssea, relativamente ao nível esperado para a idade gestacional. O rastreio baseia-se no doseamento sérico da fosfatase alcalina e fósforo. O objetivo deste estudo é avaliar a prevalência da doença metabólica óssea da prematuridade, analisar os aspetos associados a maior risco para esta doença e descrever o crescimento estaturo-ponderal dos recém-nascidos com peso ao nascer inferior a $1500 \mathrm{~g}$, com doença metabólica óssea da prematuridade.

Material e Métodos: Estudo multicêntrico, retrospetivo, observacional e descritivo em três unidades de apoio perinatal diferenciado, entre 1 de maio de 2016 e 30 de abril de 2017; foi obtida uma amostra de conveniência de recém-nascidos com muito baixo peso ao nascer. Descrevem-se as variáveis demográficas, clínicas e laboratoriais dos recém-nascidos com e sem doença metabólica óssea da prematuridade.

Resultados: Neste estudo foram incluídos 53 recém-nascidos: 30 do sexo masculino, 16 com idade gestacional $\leq 28$ semanas. Foram diagnosticados cinco casos de doença metabólica óssea da prematuridade. Neste grupo, a maioria dos doentes era do sexo masculino e apresentavam idade gestacional e peso ao nascer inferior aos do grupo sem doença metabólica óssea da prematuridade. A duração média de nutrição parentérica foi superior nos recém-nascidos com doença metabólica óssea da prematuridade e a relação cálcio/ fósforo utilizada foi inferior às recomendações nacionais. A evolução estaturo-ponderal foi semelhante nos recém-nascidos com e sem doença. Nenhum doente com doença metabólica óssea da prematuridade teve intervenção por medicina física e reabilitação.

Discussão: A prevalência de doença metabólica óssea da prematuridade foi de $9,43 \%$, valor inferior ao descrito na literatura. Contudo, apenas $50 \%$ dos recém-nascidos cumpriram o rastreio de acordo com as recomendações. Os principais fatores de risco identificados estão de acordo com a literatura.

Conclusão: A doença metabólica óssea da prematuridade é uma comorbilidade frequente nos recém-nascidos de muito baixo peso, mas encontra-se subdiagnosticada. É fundamental rastrear os recém-nascidos em risco para esta patologia, utilizando marcadores bioquímicos, assim como estruturar intervenções nutricionais e estimulação física para evitar as consequências da doença a curto e longo prazo.

Palavras-chave: Doenças Ósseas Metabólicas; Doenças do Prematuro; Estado Nutricional; Recém-Nascido de Peso Extremamente Baixo ao Nascer; Recém-Nascido Prematuro

\section{ABSTRACT}

Introduction: Metabolic bone disease of prematurity consists in a decrease of bone matrix mineral content, in comparison with the level expected for gestational age. Screening of this condition is based on serum alkaline phosphatase and phosphate levels. The aim of this study is to evaluate the prevalence of metabolic bone disease of prematurity, to assess the aspects associated with a higher risk of this disease and to describe the growth of newborns with birth weight below $1500 \mathrm{~g}$ and metabolic bone disease of prematurity. Material and Methods: Observational, retrospective, multicenter and descriptive study in three neonatal intensive care units in Portugal, from May $1^{\text {st }} 2016$ to April $30^{\text {th }}$ 2017. A convenience sample of very low birthweight newborns was obtained. Demographic, clinical, and laboratory variables were described in newborns with and without metabolic bone disease of prematurity.

Results: A total of 53 newborns were included in this study: 30 males, 16 with gestational age $\leq 28$ weeks. Five cases of metabolic bone disease of prematurity were diagnosed. In this group, the majority of patients was male and presented a lower gestational age and birth weight, in comparison with the group without metabolic bone disease of prematurity. The average duration of parenteral nutrition was higher in newborns with metabolic bone disease of prematurity and the calcium/phosphate ratio was lower than the recommended values. Growth was similar in both groups. No patient with metabolic bone disease of prematurity underwent physical rehabilitation.

Discussion: The prevalence of metabolic bone disease of prematurity was $9.43 \%$, which is lower than what is described in the literature. However, only $50 \%$ of newborns completed the screening according to the recommendations. The main risk factors identified concur with the literature.

Conclusion: Metabolic bone disease of prematurity is a frequent but underdiagnosed comorbidity in very low birthweight newborns. It is essential to screen newborns at risk for this condition, using biochemical markers, as well as structure nutritional interventions and physical stimulation in order to avoid short and long-term consequences of this disease.

1. Serviço de Pediatria. Hospital Espírito Santo de Évora. Évora. Portugal.

2. Serviço de Pediatria. Hospital Divino Espírito Santo. Ponta Delgada. Portugal.

3. Serviço de Pediatria. Hospital São Francisco Xavier. Centro Hospitalar Lisboa Ocidental. Lisboa. Portugal.

4. Serviço de Neonatologia. Hospital Espírito Santo de Évora. Évora. Portugal.

5. Serviço de Neonatologia. Hospital São Francisco Xavier. Centro Hospitalar Lisboa Ocidental. Lisboa. Portugal.

$\triangle$ Autor correspondente: Raquel Costa. raquelfreitascost@gmail.com

Recebido: 27 de junho de 2018 - Aceite: 05 de abril de 2019 | Copyright @ Ordem dos Médicos 2019 
Keywords: Bone Diseases, Metabolic; Infant, Extremely Low Birth Weight; Infant, Premature; Infant, Premature, Diseases; Nutritional Status

\section{INTRODUÇÃO}

A doença metabólica óssea da prematuridade (DMO), consiste na diminuição da matriz óssea, relativamente ao nível esperado para fetos ou crianças de tamanho/idade gestacional idênticos..$^{1-4}$

Apesar dos avanços na qualidade dos cuidados médicos, nomeadamente no suporte nutricional, a DMO mantémse uma importante comorbilidade nos recém-nascidos (RN) com muito baixo peso ao nascer (RNMBP) e nos recémnascidos com doença crónica, sobretudo patologia respiratória e gastrointestinal, com internamentos prolongados em Unidade de Cuidados Intensivos Neonatais (UCIN). ${ }^{2,5}$

A incidência exata desta patologia não é conhecida, para o que contribui a falta de consenso na definição e o reduzido número de estudos, mas estima-se que afete até $55 \%$ dos RNMBP. ${ }^{1,3}$ Não existem à data estudos de prevalência desta doença conduzidos em UCIN portuguesas, sendo por isso desconhecida a realidade nacional.

A formação do esqueleto inicia-se pela sexta semana de gestação, sendo o terceiro trimestre crítico para a mineralização óssea intra-uterina. Esta está dependente da transferência de cálcio $(\mathrm{Ca})$ e fósforo $(\mathrm{P})$ para o feto através da placenta, ${ }^{6}$ sendo o metabolismo fosfo-cálcico regulado pela ação da vitamina $\mathrm{D}$ e paratormona $(\mathrm{PTH}) \cdot{ }^{5}$ Para além da homeostasia $\mathrm{Ca} / \mathrm{P}$, também a estimulação mecânica é fundamental neste processo, uma vez que, sendo o osso um órgão, a sua formação está dependente da função. ${ }^{7}$

Os principais fatores de risco conhecidos para DMO dividem-se em fatores pré-natais, que afetam o crescimento intra-uterino do osso e em fatores pós-natais, associados a perturbação da homeostase óssea do RN. ${ }^{2}$

Os principais fatores de risco pré-natais são fatores genéticos, hábitos tabágicos maternos durante a gestação, patologia placentar, hipovitaminose $\mathrm{D}$ materna e uso de fármacos (nomeadamente sulfato de magnésio). ${ }^{3,6}$

Após o nascimento, os fatores de risco conhecidos são: idade gestacional $(I G)<28$ semanas, peso ao nascer $(P N)<1500 \mathrm{~g}$, sexo masculino, uso de fármacos metabolicamente ativos no osso usados nas UCIN (diuréticos, corticóides, metilxantinas e bicarbonato de sódio), imobilização e fatores nutricionais. ${ }^{3,6}$

A estimulação mecânica do osso do RN é inferior no ambiente extra-uterino, em relação à que se consegue no ambiente intra-uterino, através dos movimentos do RN contra a parede abdominal materna. Assim, a imobilização dos RN pré-termo constitui um importante fator de risco pósnatal para DMO e as intervenções baseadas na estimulação física regular destes doentes mostraram uma melhoria na mineralização óssea. ${ }^{7,8}$

Os fatores nutricionais pós-natais incluem atraso na introdução da alimentação entérica, cursos de nutrição parentérica prolongados, nomeadamente superiores a quatro semanas, relações $\mathrm{Ca} / \mathrm{P}$ na nutrição parentérica inadequadas $(<1,3: 1 \mathrm{em}$ molar), uso de leite materno (LM) não fortificado e défice de vitamina D.,3
Relativamente à relação $\mathrm{Ca} / \mathrm{P}$ na nutrição parentérica, apesar de esta não ser consensual, à luz da evidência científica atual, considera-se que a relação 1,3:1 (molar) será a mais adequada, por parecer associar-se a maior retenção mineral no RN pré-termo., ${ }^{3,69}$ Não obstante, nos RNMBP e sobretudo nos leves para a IG (peso < P10 para IG), nos primeiros dias de vida, a relação $\mathrm{Ca} / \mathrm{P}$ deve ser mantida entre os 0,8-1:1 (molar), de forma a evitar a hipofosfatemia precoce que pode ocorrer com a otimização do aporte calórico e proteico. ${ }^{10}$

$\mathrm{O}$ uso de LM fortificado está indicado nos RN pré-termo, nascidos com menos de $1500 \mathrm{~g}$, uma vez que o LM contém quantidade insuficiente de Ca e P para assegurar o crescimento destes RN. A fortificação deve ser iniciada quando o volume de leite materno atinge $100 \mathrm{~mL} / \mathrm{kg} / \mathrm{dia}$, devendo ser mantida até à alta hospitalar. ${ }^{11} \mathrm{~A}$ fortificação pode ser baseada numa fórmula padrão ou poderá ser personalizada, com base nas necessidades individuais de cada RN. ${ }^{3,6}$

$A$ vitamina $D$ regula o metabolismo $\mathrm{Ca} / \mathrm{P}$ e o seu défice relaciona-se com menor retenção mineral. ${ }^{5} \mathrm{De}$ acordo com as recomendações nacionais, os RN pré-termo devem ser suplementados com $800-1000$ UI de vitamina D diariamente. ${ }^{11}$

Clinicamente a DMO manifesta-se com um espectro entre a doença silenciosa e o raquitismo com fraturas ósseas, sendo algumas das manifestações tardias mais frequentes o atraso do crescimento, a má progressão ponderal e a baixa estatura. ${ }^{8}$

Sendo uma doença silenciosa, muitas vezes com manifestações apenas tardias, o rastreio da DMO está recomendado e deve ser dirigido aos RN com fatores de risco, a partir da quarta semana de vida e repetido de forma seriada (de duas em duas semanas), enquanto os RN mantiverem os referidos fatores de risco. ${ }^{3-6}$ Apenas assim se conseguirá um diagnóstico precoce e uma intervenção adequada e atempada, de forma a evitar as manifestações a longo prazo. Na doença instalada, a monitorização baseia-se nos mesmos marcadores bioquímicos. . $^{3-6,12}$

O diagnóstico precoce e a monitorização são obtidos através de marcadores bioquímicos ( $\mathrm{P}$ e fosfatase alcalina [FA]). A interpretação em conjunto dos resultados de $\mathrm{P}$ e FA, mostrou uma sensibilidade $100 \%$ e especificidade de $70 \%$ para DMO..$^{3,12,13}$ Assim, os valores FA $>900 \mathrm{UI} / \mathrm{L}$ associados a hipofosfatemia $<5,5 \mathrm{mg} / \mathrm{dL}[<1,8 \mathrm{mmol} / \mathrm{L}]$ ou FA $>600 \mathrm{UI} / \mathrm{L}$ com tendência crescente e hipofosfatemia persistente $<5,5 \mathrm{mg} / \mathrm{dL}$ nas avaliações seriadas sugerem, de forma precoce, DMO ${ }^{12,13} \mathrm{O}$ valor do Ca sérico é geralmente mantido dentro de valores normais, por ação da PTH, e neste sentido não constitui um marcador precoce de doença. ${ }^{3}$ Os marcadores imagiológicos da doença apresentam alterações tardias e não estão indicados por rotina. ${ }^{3}$

Os pilares de intervenção perante o diagnóstico tencionam corrigir os fatores de risco pós-natais, associados ao internamento em UCIN. Estes devem integrar um conjunto de intervenções nutricionais que visem melhorar o 
suprimento de Ca e P ao RN, seja na nutrição parentérica, alimentação entérica ou suplementação, cumprindo as recomendações nacionais referidas. ${ }^{8,9}$ Deve ser feito um esforço para reduzir a duração da nutrição parentérica e iniciar o mais precocemente possível a alimentação entérica, reduzindo o número de dias da sua suspensão. Adicionalmente, devem ser revistos e suspensos, se possível, os fármacos associados ao aumento do turnover ósseo. ${ }^{3,6}$ Nos cuidados diários ao RN, devem também ser incluídas estratégias de intervenção física com os benefícios já descritos. ${ }^{7}$

No presente artigo, os autores têm como objetivo avaliar a prevalência da DMO e descrever os aspetos associados a risco aumentado para esta doença. Adicionalmente, pretende-se descrever o crescimento estaturo-ponderal dos $\mathrm{RN}$ com peso ao nascer (PN) inferior a $1500 \mathrm{~g}$ com DMO.

\section{MATERIAL E MÉTODOS}

Estudo retrospetivo multicêntrico observacional descritivo, conduzido em três unidades de apoio perinatal diferenciado, entre 1 de maio de 2016 e 30 de abril de 2017, tendo sido estudada uma amostra de conveniência não aleatorizada, de RN prematuros com peso inferior a $1500 \mathrm{~g}$. Foram excluídos os doentes falecidos, transferidos para outras Unidades de Cuidados Intensivos Neonatais (UCIN) e aqueles sem seguimento após alta hospitalar nas UCIN de realização do estudo. Foram ainda excluídos os RN com malformações congénitas, doenças neuromusculares, erros inatos do metabolismo e outras doenças ósseas.

Foram avaliadas características sociodemográficas, hábitos tabágicos durante a gravidez, patologia placentar, idade gestacional e peso ao nascer. Os fatores de risco estudados incluíram o uso de fármacos metabolicamente ativos no osso (corticóides, diuréticos, metilxantinas e bicarbonato de sódio) e fatores nutricionais: número de dias de nutrição parentérica; relação $\mathrm{Ca} / \mathrm{P}$ na alimentação parentérica9; dias de pausa na nutrição entérica; fortificação do LM e dose de vitamina $D$ administrada. ${ }^{11}$ Adicionalmente foram avaliadas intervenções terapêuticas por Medicina Física e de Reabilitação (MFR). Este estudo retrospetivo baseia-se na prática clínica, e neste sentido, não foi possível estudar a hipovitaminose D materna, por esta não ser avaliada de forma sistemática nas grávidas.

$O$ rastreio da doença está recomendado e baseia-se em determinações seriadas de FA e P. A primeira avaliação destes parâmetros bioquímicos deve ser realizada às quatro semanas de idade cronológica do $\mathrm{RN}$ e repetida de duas em duas semanas. O diagnóstico de DMO foi estabelecido com base nos marcadores bioquímicos com maior sensibilidade e especificidade na literatura: FA > $900 \mathrm{UI} / \mathrm{L}$ e $\mathrm{P}<5,5 \mathrm{mg} / \mathrm{dL}$ [ $<1,8 \mathrm{mmol} / \mathrm{L}]$, ou FA $>600 \mathrm{UI} / \mathrm{L}$ com tendência crescente e hipofosfatemia $<5,5 \mathrm{mg} / \mathrm{dL}$ persistente em avaliações seriadas. 3,12,13

Para a avaliação do crescimento estaturo-ponderal dos RN avaliaram-se os dados antropométricos (peso e comprimento) ao longo dos 12 meses em que o estudo decorreu. O cálculo do z-score foi realizado com base na idade corrigida (curvas de crescimento de Fenton até as 50 semanas de idade pós-menstrual e curvas de crescimento da Organização Mundial de Saúde a partir desta idade). ${ }^{14,15}$ Os dados do peso e comprimento obtidos foram avaliados de forma continuada na UCIN durante o internamento, até à data de alta. Após a alta, foram recolhidos os dados de crescimento disponíveis na consulta de Neonatologia.

A análise estatística foi realizada com recurso ao software SPSS versão $23^{\circledR}$, procedendo-se a uma análise descritiva para caracterização dos doentes, das variáveis conhecidas como fatores de risco e do crescimento estaturo-ponderal dos doentes. Foi obtida aprovação das comissões de ética prévia ao início do estudo. O estudo apresentado neste artigo está incluído num projeto de investigação que engloba, para além de uma análise retrospetiva cujos dados foram colhidos de forma anónima, um estudo prospetivo. Após avaliação nas comissões de ética nos três hospitais onde o estudo foi conduzido, foi exigido consentimento informado aos pais dos recém-nascidos incluídos no estudo prospetivo.

\section{RESULTADOS}

Durante o período de estudo, nasceram $83 \mathrm{RN}$ com $\mathrm{PN}<1500 \mathrm{~g}$. Destes, foram excluídos 30 (nove óbitos, 11 sem seguimento nos hospitais participantes e 10 transferidos para outras UCIN). Os doentes excluídos apresentavam características semelhantes, quanto ao peso ao nascer e idade gestacional.

Foram incluídos 53 doentes, a maioria do sexo masculino e com idade gestacional média de $29 \pm 2,7$ semanas (idade gestacional mínima de 23 semanas e máxima de 34 semanas). A maioria dos doentes incluídos apresentava idade gestacional compreendida entre as 29 e as 32 semanas (49\%). O peso ao nascer médio foi de 1118,6 g $\pm 261,3 \mathrm{~g}$ (peso mínimo de $625 \mathrm{~g}$ e máximo de $1491 \mathrm{~g})$. A caracterização dos doentes incluídos encontra-se descrita na Tabela 1.

Com base nos marcadores bioquímicos referidos (doseamentos séricos seriados de $P$ e FA), foram

Tabela 1 - Caracterização dos doentes incluídos ( $n=53)$, quanto à idade gestacional, peso ao nascer, comprimento e perímetro cefálico

\begin{tabular}{ll}
\hline Doentes incluídos & $\mathrm{n}=53$ \\
\hline Masculino (n) (\%) & $30(56,6 \%)$ \\
Idade gestacional (semanas) & \\
Média \pm Desvio padrão [Mínimo; Máximo] & $29 \pm 2,7[23 ; 34]$ \\
Idade gestacional (semanas) & \\
$\leq 28$ semanas & $16(30,2 \%)$ \\
$29-32$ semanas & $26(49,0 \%)$ \\
$\geq 32$ semanas & $11(20,8 \%)$ \\
Peso ao nascer (gramas) & \\
Média \pm Desvio padrão & $1118,6 \pm 261,3$ \\
Mediana [Mínimo; Máximo] & $1148[625 ; 1491]$ \\
Comprimento ao nascer (centímetros) & \\
Média \pm Desvio padrão & $36,7 \pm 3,49$ \\
Mediana [Mínimo; Máximo] & 37,$5 ;[29,0 ; 46,5]$ \\
Perímetro cefálico ao nascer (centímetros) & \\
Média \pm Desvio padrão & $26,2 \pm 2,35$ \\
Mediana [Mínimo; Máximo] & 26,$45 ;[21,2 ; 30,5]$ \\
\hline
\end{tabular}


diagnosticados cinco casos de DMO (9,43\%). Contudo, verificou-se que apenas em 15 dos $53 \mathrm{RN}$, foi realizado por rotina o doseamento de $\mathrm{FA}$ e $\mathrm{P}$ às quatro semanas de idade cronológica e mantiveram as avaliações analíticas seriadas apenas $10 \mathrm{RN}$ até à alta clínica. Assim, nos RNMBP que cumpriram o rastreio, a incidência de DMO foi de $50 \%$.

$\mathrm{Na}$ análise dos fatores associados a maior risco para DMO (Tabela 2), foram identificados com maior frequência doentes do sexo masculino. O PN e a IG foram, em média, inferiores no grupo de doentes com $\mathrm{DMO}$, relativamente ao grupo sem doença. Ainda relativamente aos fatores de risco pré-natais, foram identificados com maior frequência patologia placentar e hábitos tabágicos durante a gravidez nos doentes sem DMO.

Dos aspetos nutricionais mais frequentemente implicados na doença, o número de dias de nutrição parentérica foi, em média, superior nos RN com DMO (24,8 dias $\pm 9,4$ dias vs 17,7 dias $\pm 8,9$ dias). O número de dias de pausa de alimentação entérica foi superior no grupo sem DMO (2,5 dias $\pm 2,9$ dias vs 0,8 dias $\pm 0,21$ dias). A relação $\mathrm{Ca} / \mathrm{P}$ utilizada na nutrição parentérica foi inferior à recomendada (< 1,3:1 em molar) no grupo de doentes identificados com doença. A maioria dos RN fez aleitamento materno, e este foi sempre fortificado e/ou suplementado de acordo com as recomendações nacionais. ${ }^{11}$ A fortificação/suplementação foi baseada em fórmula padrão e/ou personalizada pelo serviço de nutrição hospitalar. A suplementação com vitamina $D$ esteve também de acordo com as recomendações nacionais nos dois grupos. ${ }^{11}$

Quanto aos fármacos metabolicamente ativos no osso, em nenhum RN foi utilizado bicarbonato de sódio e em todos os doentes foi usado citrato de cafeína de acordo com as recomendações nacionais para prevenção de apneia da prematuridade. Foram utilizados diuréticos em $60 \%$ dos RN com DMO (Tabela 2 ).

Salienta-se que nenhum doente com o diagnóstico de DMO fez estimulação física como intervenção terapêutica (Tabela 2).

Nesta amostra, de uma forma global, os RN apresentaram uma evolução estaturo-ponderal semelhante nos dois grupos, com z-score negativo (Tabela 3). Em média, tanto para a variável peso como para a variável comprimento, o z-score foi inferior no grupo com DMO (peso: $-1,29 \pm 1,0$ vs $-1,37 \pm 0,92$; comprimento: $-1,30 \pm 2,32$ vs $-1,69 \pm 2,48)$.

\section{DISCUSSÃO}

A DMO mantém-se uma importante comorbilidade nos RNMBP e nos RN com doença crónica, sobretudo naqueles com internamentos prolongados em UCIN.

Tabela 2 - Caracterização dos fatores de risco para DMO

\begin{tabular}{|c|c|c|}
\hline & $\begin{array}{l}\text { Grupo sem DMO } \\
(n=48)\end{array}$ & $\begin{array}{l}\text { Grupo com DMO } \\
(n=5)\end{array}$ \\
\hline $\begin{array}{l}\text { Distribuição género }(\mathbf{n}) \\
\text { Masculino } \\
\text { Feminino }\end{array}$ & $\begin{array}{l}26(54,1 \%) \\
22(45,8 \%)\end{array}$ & $\begin{array}{l}4(80 \%) \\
1(20 \%)\end{array}$ \\
\hline $\begin{array}{l}\text { Idade gestacional (semanas) } \\
\text { Média } \pm \text { Desvio padrão }\end{array}$ & $29,5 \pm 2,7$ & $27,4 \pm 2,3$ \\
\hline $\begin{array}{l}\text { Peso ao nascer (gramas) } \\
\text { Média } \pm \text { Desvio padrão } \\
\text { Mediana [Mínimo; Máximo] }\end{array}$ & $\begin{array}{l}1137,1 \pm 262,5 \\
1197,5[660,0 ; 1491,0)\end{array}$ & $\begin{array}{l}941,4 \pm 185,7 \\
967,0[625,0 ; 1086,0]\end{array}$ \\
\hline Mãe fumadora (n) (\%) & $5(10,4 \%)$ & 0 \\
\hline Placenta patológica (n) (\%) & $11(20 \%)$ & 0 \\
\hline $\begin{array}{l}\text { Fármacos (n) (\%) } \\
\text { - Diuréticos } \\
\text { - Corticóides }\end{array}$ & $\begin{array}{l}15(34,9 \%) \\
15 \\
8\end{array}$ & $\begin{array}{l}3(60 \%) \\
3 \\
1\end{array}$ \\
\hline $\begin{array}{l}N^{\circ} \text { de dias de parentérica (dias) } \\
\text { Média } \pm \text { Desvio padrão }\end{array}$ & $17,7 \pm 8,9$ & $24,8 \pm 9,4$ \\
\hline $\begin{array}{l}\text { Relação } \mathrm{Ca}^{2+} / \mathbf{P} \text { (molar) na nutrição parentérica } \\
\text { Média } \pm \text { Desvio padrão }\end{array}$ & $1,3 \pm 0,3$ & $1,04 \pm 0,5$ \\
\hline $\begin{array}{l}\text { Pausa alimentação entérica (dias) } \\
\text { Média } \pm \text { Desvio padrão }\end{array}$ & $2,5 \pm 2,9$ & $0,8 \pm 0,21$ \\
\hline $\begin{array}{l}\text { Aleitamento materno (n) (\%) } \\
\text { Fórmula padrão } \\
\text { Suplementação personalizada }\end{array}$ & $\begin{array}{l}34(70,8 \%) \\
30(62,5 \%) \\
14(29,1 \%)\end{array}$ & $\begin{array}{l}5(100 \%) \\
5(100 \%) \\
3(60 \%)\end{array}$ \\
\hline $\begin{array}{l}\text { Vitamina D (Ul/dia) } \\
\text { Média } \pm \text { Desvio padrão }\end{array}$ & $902,6 \pm 153,4$ & 1000 \\
\hline Intervenções por MFR (n) & 4 & 0 \\
\hline
\end{tabular}

Caracterização dos doentes quanto aos fatores de risco conhecidos para DMO: sexo; idade gestacional; peso ao nascer; hábitos tabágicos durante a gravidez; patologia placentar; uso de fármacos metabolicamente ativos no osso*; $n^{\circ}$ de dias de nutrição parentérica; relação $\mathrm{Ca} / \mathrm{P}$ (molar) na nutrição parentérica; fortificação do leite materno; dose de suplementação com vitamina D; e intervenções por medicina física e reabilitação (MFR).

*Em nenhum doente foi usado bicarbonato de sódio. Todos os doentes foram tratados com citrato de cafeína. 
Tabela 3 - Evolução do peso e comprimento dos doentes com e sem DMO

\begin{tabular}{|c|c|c|c|}
\hline & & $\begin{array}{l}\text { Grupo sem DMO } \\
(n=48)\end{array}$ & $\begin{array}{l}\text { Grupo com DMO } \\
(n=5)\end{array}$ \\
\hline \multirow{4}{*}{ Peso } & Gramas & & \\
\hline & Média \pm Desvio padrão & $2526 \pm 1765$ & $2189 \pm 1397$ \\
\hline & $z$-score* & & \\
\hline & Média \pm Desvio padrão & $-1,29 \pm 1,0$ & $-1,37 \pm 0,92$ \\
\hline \multirow{4}{*}{ Comprimento } & Centímetros & & \\
\hline & Média \pm Desvio padrão & $49,93 \pm 10,13$ & $47,48 \pm 8,23$ \\
\hline & z-score* & & \\
\hline & Média \pm Desvio padrão & $-1,30 \pm 2,32$ & $-1,69 \pm 2,48$ \\
\hline
\end{tabular}

* Cálculo do z-score realizado com base na idade corrigida (curvas de crescimento de Fenton até às 50 semanas de idade pós-menstrual e curvas de crescimento da Organização Mundial de Saúde a partir desta idade).

A prevalência de DMO encontrada neste estudo foi de $9,43 \%$. Este resultado é muito inferior ao descrito na literatura, que estima que esta doença afete mais de metade dos RNMBP. ${ }^{1-3,5}$ A prevalência reduzida é justificada, em parte, nesta amostra, pela ausência de um rastreio bioquímico sistemático dos RN com fatores de risco e sugere um subdiagnóstico de DMO nas UCIN que participaram no estudo. De facto, quando consideramos o número de $\mathrm{RN}$ aos quais foi realizado o rastreio de acordo as recomendações, os diagnósticos de DMO correspondem a $50 \%$ dos RN. O elevado número de doentes excluídos, pode ter também contribuído para o reduzido número de diagnósticos de $\mathrm{DMO}$, em particular nos $\mathrm{RN}$ com patologia gastrointestinal e necessidade de transferência para intervenção especializada em centros cirúrgicos. Este subgrupo de doentes tem importantes fatores de risco nutricionais, nomeadamente cursos de nutrição parentérica prolongados e pausas prolongadas na alimentação entérica. ${ }^{3-6}$

O sexo masculino, a IG e PN inferiores estão descritos na literatura em associação com $\mathrm{DMO},{ }^{3-6}$ tendo estas características sido identificadas com maior frequência na nossa amostra. A frequência de mães fumadoras e com patologia placentar foi superior no grupo de RN sem DMO, contrariamente ao descrito na literatura, assim como o número de dias de pausa de alimentação entérica. ${ }^{3-6}$ Os autores admitem que estes fatores de risco não foram identificados com maior frequência no subgrupo de doentes com DMO pela reduzida amostra de doentes estudada.

Os aspetos relacionados com a nutrição dos RN são um dos pilares fisiopatológicos da doença, com um importante papel na sua instalação e manutenção. Estes cuidados são um dos pontos críticos da intervenção, para prevenção e tratamento de DMO. De facto, nesta amostra, o número de dias de nutrição parentérica foi superior nos RN com DMO e a relação $\mathrm{Ca} / \mathrm{P}$ utilizada foi, neste grupo, inferior à recomendada a nível nacional ( $<1,3: 1$ molar). ${ }^{9}$ Foram respeitadas as recomendações nacionais quanto à fortificação do leite materno e suplementação com vitamina D. ${ }^{11}$

Paralelamente a estratégias nutricionais adequadas, as intervenções de MFR são fundamentais na prevenção e no controlo da progressão da doença, ${ }^{3,7,16,17}$ no entanto, nenhum doente com DMO foi submetido a estimulação motora com este propósito.
Nos três RN com DMO e que se encontravam medicados com fármacos associados a maior turnover ósseo, a sua suspensão não foi possível pela importância no tratamento de comorbilidades (neste caso a displasia broncopulmonar). No entanto, a identificação da doença conduziu à substituição da fortificação do LM com fórmula padrão por uma suplementação personalizada, baseada em necessidades minerais mais elevadas nestes RN.

A avaliação dos dados da somatometria é um ponto-chave na avaliação do crescimento de RN internados nas UCIN. A repercussão da DMO no crescimento é uma das manifestações importantes da doença. ${ }^{18}$ Nesta amostra, em média, a evolução do peso e comprimento em ambos os grupos, documenta valores de z-score inferiores nos doentes com DMO. Porém, o número de RN com diagnóstico de $\mathrm{DMO}$ é reduzido e a duração do período de seguimento, inferior a 12 meses, é muito curta para avaliação dos efeitos da doença sobre o crescimento destes doentes.

Este estudo é limitado pelo reduzido número de doentes incluídos, numa amostra de conveniência, não representativa da população. A participação de outras UCIN, nomeadamente as que funcionam como centros cirúrgicos de patologia abdominal e que recebem $\mathrm{RN}$ com múltiplos fatores de risco, traduzir-se-ia num maior número de doentes incluídos. A aplicação de um rastreio sistemático poderia estimar uma prevalência mais aproximada dos valores descritos na literatura ${ }^{1-3,5}$ com maior conhecimento sobre a realidade nacional da $\mathrm{DMO}$. O reduzido número de doentes com diagnóstico de DMO limita as conclusões a tirar na caracterização dos fatores de risco. A duração curta do período de estudo é outra limitação. Assim, apenas a avaliação continuada de dados antropométricos, permitirá clarificar os efeitos da DMO no crescimento destes RN.

\section{CONCLUSÃO}

A DMO é frequente em RN internados nas UCIN mas pouco discutida a nível nacional. Os resultados obtidos nesta amostra, sugerem que a DMO é subdiagnosticada, não traduzindo a real prevalência da doença. Deve ser realizado um rastreio sistemático em RNMBP, utilizando marcadores bioquímicos de custo económico reduzido, como ponto de partida para o seu diagnóstico. ${ }^{19}$ Existe a necessidade 
de realizar estudos adicionais com uma amostra representativa, abrangendo um maior número de UCIN.

A identificação precoce da doença é fundamental para assegurar intervenções atempadas. Das estratégias disponíveis na abordagem da DMO, as intervenções nutricionais e a estimulação física destacam-se como os pilares do controlo da doença e devem integrar a rotina de cuidados aos RN. ${ }^{20}$ A monitorização laboratorial e as atitudes de intervenção devem ser mantidas mesmo após a alta, na doença instalada, ou enquanto se mantiverem os fatores de risco. A avaliação das manifestações clínicas tardias deve ser atentamente acompanhada nas consultas de Neonatologia, pelo impacto negativo na saúde destes doentes.

\section{AGRADECIMENTOS}

Unidades de Neonatologia, Serviços de Nutrição e Serviços de Medicina Física e Reabilitação do Hospital Espírito Santo de Évora, Hospital São Francisco Xavier e Hospital Divino Espírito Santo de Ponta Delgada.

\section{REFERÊNCIAS}

1. Choban $\mathrm{P}$, Dickerson $\mathrm{R}$, Malone $\mathrm{A}$, Worthington $\mathrm{P}$, Compher $\mathrm{C}$ A.S.P.E.N. Clinical Guidelines. J Parenter Enter Nutr. 2013;37:714-44.

2. Bozzetti $\vee$, Tagliabue P. Metabolic bone disease in preterm newborn: an update on nutritional issues. Ital J Pediatr. 2009;35:20.

3. Nallagonda S, Nallagonda M, Deorukhkar A. Metabolic bone disease of prematurity — an overview. Paediatr Child Heal. 2017;27:14-7.

4. Manfredini VA. Metabolic bone disease of prematurity: a review of minerals supplementation and disease monitoring. J Neonatal Biol. 2015;4:1-4.

5. Rustico SE, Calabria AC, Garber SJ. Metabolic bone disease of prematurity. J Clin TransI Endocrinol. 2014;1:85-91.

6. Rehman MU. Metabolic bone disease in the preterm infant: current state and future directions. World J Methodol. 2015;5:115.

7. Land C, Schoenau E. Fetal and postnatal bone development: reviewing the role of mechanical stimuli and nutrition. Best Pract Res Clin Endocrinol Metab. 2008;22:107-18.

8. Machado A, Rocha G, Silva Isabel A, Alegrete N, Guimarães H. Bone fractures in a neonatal intensive care unit. Acta Med Port. 2015;28:204.

9. Secção de Neonatologia da SPP. Nutrição parentérica no recémnascido: $1^{\text {a }}$ revisão do Consenso Nacional, 2008. Acta Pediatr Port. 2008;39:125-34

10. Mihatsch W, Fewtrell M, Goulet $O$, Molgaard C, Picaud JC, Senter T. ESPGHAN/ESPEN/ESPR/CSPEN guidelines on pediatric parenteral nutrition: calcium, phosphorus and magnesium. Clin Nutr. 2018;37:2360-5

11. Macedo I, Alexandrino AM, Pissarra S, Cardoso M. Nutrição entérica na

\section{PROTEÇÃO DE PESSOAS E ANIMAIS}

Os autores declaram ter seguido os protocolos do seu centro de trabalho acerca da publicação de dados e que os procedimentos seguidos estavam de acordo com os regulamentos estabelecidos pelos responsáveis da Comissão de Investigação Clínica e Ética e de acordo com a Declaração de Helsínquia da Associação Médica Mundial.

\section{CONFIDENCIALIDADE DOS DADOS}

Os autores declaram ter seguido os protocolos do seu centro de trabalho acerca da publicação de dados.

\section{CONFLITO DE INTERESSES}

Os autores declaram que não existem quaisquer conflitos de interesse associados a esta publicação que possam ter influenciado os resultados obtidos.

\section{FONTES DE FINANCIAMENTO}

Estudo realizado com o apoio da Sociedade Portuguesa de Neonatologia (Bolsa Milupa DN-ELN).

criança nascida pré-termo : revisão do consenso nacional. Acta Pediatr Port. 2014;45:326-39.

12. Harrison CM, Johnson K, McKechnie E. Osteopenia of prematurity: a national survey and review of practice. Acta Paediatr. 2008;97:407-13.

13. Abdallah EA, Said RN, Mosallam DS, Moawad EM, Kamal NM, Fathallah MG. Serial serum alkaline phosphatase as an early biomarker for osteopenia of prematurity. Medicine. 2016;95:1-5.

14. Fenton TR, Kim JH. A systematic review and meta-analysis to revise the Fenton growth chart for preterm infants. BMC Pediatrics.2013;13:59.

15. World Health Organization. Child growth standards. [consultado 2018 jan 20]. Disponível em: http://www.who.int/childgrowth/en/.

16. Rigo J, Pieltain C, Salle B, Senterre J. Enteral calcium, phosphate and vitamin $\mathrm{D}$ requirements and bone mineralization in preterm infants. Acta Paediatr. 2007;96:969-74.

17. Rauch F, Schoenau E. Skeletal development in premature infants: a review of bone physiology beyond nutritional aspects. Arch Dis Child Fetal Neonatal Ed. 2002;86:F82-5.

18. Embleton N, Wood CL. Growth, bone health, and later outcomes in infants born preterm. J Pediatr. 2014;90:529-32.

19. Ramón AM, Espuelas CF, Calmarza PC, Gracia SR, Del Cacho MJ. Factores de riesgo y marcadores bioquímicos de la enfermedad metabólica ósea del recién nacido prematuro. Rev Chil Pediatr. 2017;88:487-94

20. Stalnaker KA, Poskey GA. Osteopenia of prematurity: does physical activity improve bone mineralization in preterm infants? Neonatal Netw. 2016;35:95-104. 